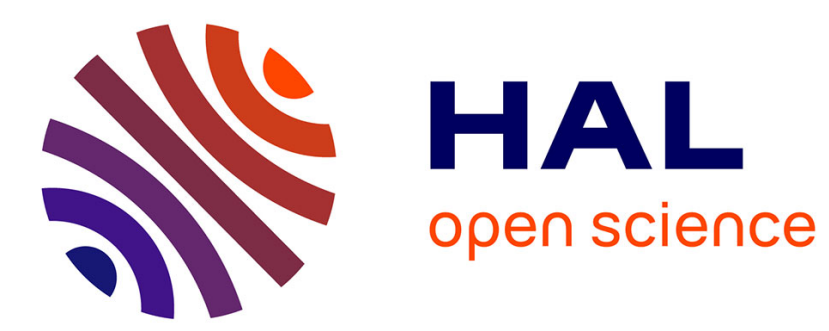

\title{
L'émigration tunisienne en 1972
}

Gildas Simon

\section{To cite this version:}

Gildas Simon. L'émigration tunisienne en 1972. Méditerranée : revue géographique des pays méditerranéens, 1973, 4, pp.95-109. halshs-00822846

\section{HAL Id: halshs-00822846 https://shs.hal.science/halshs-00822846}

Submitted on 15 May 2013

HAL is a multi-disciplinary open access archive for the deposit and dissemination of scientific research documents, whether they are published or not. The documents may come from teaching and research institutions in France or abroad, or from public or private research centers.
L'archive ouverte pluridisciplinaire HAL, est destinée au dépôt et à la diffusion de documents scientifiques de niveau recherche, publiés ou non, émanant des établissements d'enseignement et de recherche français ou étrangers, des laboratoires publics ou privés. 


\title{
L’EMIGRATION TUNISIENNE EN 1972
}

\author{
Gildas Simon $^{1}$
}

\begin{abstract}
SUMMARY - Since 1955, Tunisia has experienced a relatively high level of emigration (ie. 200000 emigrants for a population of 5 millions). This article studies the beginnings of this movement whose development is due to several factors : The internal and external labour situation, changing aspirations of the younger generations etc ... The characterisfics of this movement have evolued rapidly over a fifteen year period : administrative intervention, raising if professional qualification, family departures, development of urban emigration etc ... The consequences of this emigration are importants for the national economy (inflow of currency) but the impact at the local level varies considerably from region to region.
\end{abstract}

RESUME - La Tunisie connaît depuis 1955 un mouvement d'émigration qui a pris une certaine ampleur (200 000 émigrés .pour une population de 5 millions). Cette étude explique le déc1enchement de ce mouvement dont la poursuite est due à plusieurs facteurs : le marché intérieur et extérieur de l'emploi, les aspirations nouvelles des jeunes générations, etc ... Les caractères de ce mouvement ont évolué rapidement depuis une quinzaine d'années : intervention de l'administration, amélioration de la qualification professionnelle, départ des familles, développement de l'émigration urbaine, etc ... Les conséquences de cette émigration sont importantes pour l'économie nationale (rentrées de devises) mais l'impact au plan local varie fortement d'une région à l'autre.

La Tunisie s'est intégrée plus tardivement que l'Algérie et le Maroc dans le grand mouvement migratoire qui draine les travailleurs des pays méditerranéens vers les foyers industriels et les agglomérations urbaines de l'Europe Occidentale. De ce fait, mais en raison aussi de la personnalité même de la Tunisie, de son évolution économique et psychosociale depuis l'Indépendance, l'émigration tunisienne présente-t-elle des caractères spécifiques que l'on s'efforcera de dégager tout au long de cette étude (I).

\section{I - LE DEVELOPPEMENT RECENT DE L'EMIGRATION TUNISIENNE}

\section{A - L'EVOLUTION HISTORIQUE}

L'émigration temporaire de la main-d'oeuvre tunisienne à destination de l'Europe Occidentale et de la Libye, est un phénomène récent, qui débute en 1956 et ne prend une certaine ampleur que depuis 1963-1964.

\footnotetext{
${ }^{1}$ Maître assistant de Géographie, Université de Poitiers
} 
Certes, il existait auparavant quelques courants migratoires qui avaient entraîné l'installation de Tunisiens en Egypte au Caire et à Alexandrie depuis le XVIIIème siècle, en Libye à Tripoli, et en Algérie depuis le début du XXème siècle (en Algérie Orientale et à Alger). Mais il s'agissait surtout d'une émigration commerçante, pratiquée notamment par les Jerbiens et par les marchands de beignets de Ghomrassen. L'évolution de la situation politique et militaire, la nature des régimes économiques, les difficultés de transfert ont déterminé le départ de la majorité de ces commerçants qui sont revenus en Tunisie ou qui sont repartis s'installer en France, dans les quartiers des grandes agglomérations habités par les travailleurs maghrébins (Marseille, Lyon et Paris).

Actuellement, le nombre de Tunisiens résidant en Algérie ne dépassait pas 5000 à 7000 selon les estimations des Autorités Consulaires.

L'émigration ouvrière vers la France prend naissance en 1956 mais jusqu'en 1963-1964, elle reste très inférieure à l'émigration des Israëlites tunisiens, qui partent par familles entières et qui vont s'installer en France ou en Israël, via la France. La moitié d'entre-eux (40 000) réside en Israël et les autres sont en France, où certains ont acquis la nationalité française par voie de naturalisation (15000 environ) . Depuis 1964, l'émigration temporaire des travailleurs tunisiens est devenue le fait essentiel de ce mouvement migratoire dont les débouchés se sont diversifiés, puisque 1es flux migratoires ne concernent plus seulement la France mais aussi d'autres pays de l'Europe occidentale en recherche de main-d'oeuvre (République Fédérale Allemande, Belgique, Pays-Bas, Suisse, Autriche) ainsi que la Libye. Du fait de son développement récent, cette migration ouvrière ne possède pas encore de tradition migratoire comparable à celle de deux autres pays du Maghreb (Algérie et Maroc) où le phénomène a débuté depuis deux ou trois générations ; ceci explique certaines difficultés quant à l'insertion des Tunisiens sur les marchés du travail étrangers.

L'évolution des soldes migratoires calculés d'après le nombre de passages de Tunisiens aux frontières montre bien l'expansion croissante, bien que syncopée, de ce mouvement migratoire. Le solde dépasse le cap des 10000 en 1964, atteint 27400 en 1969, descend à 21900 en 1970 et connaît un nouveau record en 1971 (32 400). Le solde migratoire total enregistré depuis 1955 s'élève au nombre de 201000 personnes. Ce chiffre est vérifié et confirmé par les statistiques des pays d'immigration .

Au début de 1972, la répartition par pays peut être établie ainsi:

$\begin{array}{llll}\text { - } & \text { France } & : & 110000 \text { (96 821 adultes au 1.1.1971) (Ministère de l'Intér.) } \\ \text { - } & \text { R.F.A. } & : & 10000 \text { (9 890 adultes au 30.6.1971) } \\ \text { - } & \text { Libye } & : & 20000 \text { (estimation) } \\ \text { - } & \text { Algérie } & : & 7000 \text { (estimation) } \\ \text { - } & \text { Pays européens : } & 3000 \text { (Pays-Bas, Belgique, Suisse, Autriche, Scandinavie) } \\ \text { TAL } & & 150000\end{array}$

La différence avec le solde migratoire tota1 (200 000) correspond à l'émigration israëlite en Israël et aux naturalisations intervenues en France .

\section{B - PRESSION INTERNE ET APPEL EXTERIEUR}

Le mouvement migratoire dont le poids humain n'est pas négligeable (1/5 de l'accroissement naturel tunisien actuel) s'explique autant par l'évolution de la Tunisie depuis 1956 que par l'attraction et les besoins en main d'œuvre des pays étrangers. 
De nombreuses enquêtes effectuées auprès des ouvriers tunisiens à l'étranger montrent que c'est la situation de l'emploi en Tunisie qui a été et qui demeure le moteur essentiel de l'émigration temporaire des travailleurs. Les études précédentes ont prouvé la gravité et l'acuité du chômage et du sous-emploi malgré les efforts d'industrialisation et le développement des activités de service enregistrées depuis l'indépendance nationale.

Mais la ponction migratoire sur le marché de l'emploi s'effectue selon certains critères en fonction du caractère sélectif de ce type de mouvement migratoire, tant sur le plan démographique et professionnel que sur le plan sociologique, mais aussi, en raison du contrôle du mouvement migratoire par l'O.F.P.E. depuis 1968-1969. Deux aspects de cette question doivent être signalés dès à présent :

- le départ croissant des jeunes à la recherche d'un premier emploi et, notamment, ceux qui sortent des centres de formation professionnelle et ne trouvent pas de débouchés en Tunisie;

- l'émigration des ouvriers spécialisés ou qualifiés possédant une certaine expérience professionnelle (bâtiment et travaux publics, métallurgie, textile, hôtellerie). Cette main-d'oeuvre devient majoritaire pour trois raisons : les besoins des pays industrialisés, l'amélioration de la formation professionnelle en Tunisie, le départ des ouvriers qualifiés abandonnant leur emploi dans les entreprises nationales pour aller chercher en Europe des rémunérations plus élevées et pour tenter d'y connaître une certaine promotion professionnelle. Mais on ne peut plus aujourd'hui réduire l'émigration au problème du sous-emploi ; il faut en effet, le replacer dans le cadre de l'évolution considérable des mentalités et des comportements que connaît la Tunisie depuis l'indépendance sous l'influence de la révolution scolaire, du développement des mass média, de l'accroissement de la mobilité interne mais aussi externe de la population, des effets de démonstration sociale du tourisme et de l'émigration ellemême. Les aspirations de consommations, les besoins tendent à s'aligner sur ceux des pays industrialisés alors que les revenus et les rémunérations restent encore très bas. Entre les deux termes, le divorce va croissant et tend de plus en plus à impulser le mouvement migratoire.

A cette pression migratoire de la Tunisie actuelle, correspond inversement un appel en maind'oeuvre de la part des pays industrialisés européens, qui ont connu une expansion économique rapide depuis 1945. La main-d'œuvre européenne tend, on le sait, à abandonner les emplois manuels les moins rémunérés et les plus dangereux et le remplacement s'effectue généralement par des travailleurs étrangers.

L'appel du marché français a été le plus puissant, non seulement en fonction des liens qui unissent traditionnellement la Tunisie et la France mais aussi à cause de l'évolution des flux d'immigration propre à ce pays.

En raison de l'arrêt ou du ralentissement de l'immigration polonaise, belge et italienne, la France a été obligée de recourir à de nouvelles sources de main-d'oeuvre (Espagne, Portugal, Yougoslavie, Turquie) et de faire appel plus largement aux travailleurs maghrébins. La part de la population nord-africaine résidant en France est ainsi passée de 4 \% des étrangers en 1930 à $32 \%$ en 1972 (2). C'est dans ce contexte que s'est développée spontanément à partir de 1956 l'émigration tunisienne vers ce pays. Les mêmes besoins en main-d'oeuvre se manifestent 
aussi en Allemagne occidentale, aux Pays-Bas et en Libye. La situation de l'emploi dans ce dernier pays est assez particulière car ce pays en voie de développement est obligé de faire appel à des travailleurs étrangers afin de répondre aux besoins créés par le boom pétrolier et par ses conséquences économiques (travaux publics, construction, chantiers de pétrole, et même agriculture) . Cette situation a entraîné le déclenchement d'une véritable explosion migratoire dès 1966-1967, à partir des régions du Sud et du Centre. Des milliers d'émigrants souvent clandestins affluèrent aux frontières à un point tel que les autorités durent freiner ce mouvement.

\section{II - LES CARACTERES DE L'EMIGRATION ACTUELLE D'ORIGINE}

\section{A - L'ORGANISATION DU MOUVEMENT MIGRATOIRE}

L'intensité de ces courants migratoires spontanés, leur importance pour la Tunisie sur le plan de l'emploi et des rentrées en devises, ont amené la conclusion d'accords bi-latéraux de maind'oeuvre destinés à régulariser, à organiser et à planifier les mouvements de main-d'oeuvre. Ainsi furent conclus successivement l'accord avec la France en 1963 qui fut effectivement appliqué en 1969, avec la Libye en 1971, l'Allemagne Fédérale en 1969, les Pays-Bas, l'Autriche en 1971. Des missions étrangères de recrutement sont venues s'installer en Tunisie.

Parallèlement, l'O.F.P.E. recevait la charge d'organiser et de développer l'émigration en installant dans toutes les régions un dispositif efficace. Cette invention de l'administration et la collaboration avec 1es missions étrangères a modifié très sensiblement les caractères de l'émigration et a substitué aux flux spontanés un mouvement contrôlé voire sélectionné et planifié.

Mais cette intervention officielle varie selon le type de contrat de travail. Elle est très importante dans le cas d'une offre anonyme pour laquelle l'O.F.P.E. détermine une présélection et une sélection médicale, professionnelle et effectue d'autorité la répartition des partants . C'est le cas général de l'émigration dirigée vers l'Allemagne Fédérale et les autres pays européens, ainsi que vers la Libye, le rôle de l'administration est évidemment plus limité en cas d'une offre nominative et ne s'exerce alors que sur le plan médical, familial et sur la situation de l 'emploi précédent.

Tel est le cas de l'émigration en France en 1970 et en 1971. Il faut noter qu'en outre, près de 2000 travailleurs ont émigré sans contrat, en 1971, dans ce pays, où ils ont réussi à faire régulariser leur situation. On peut donc affirmer que l'émigration vers la France reste un mouvement spontané bien que contrôlé, alors que les mouvements dirigés vers les autres pays sont organisés et planifiés par l'administration. 


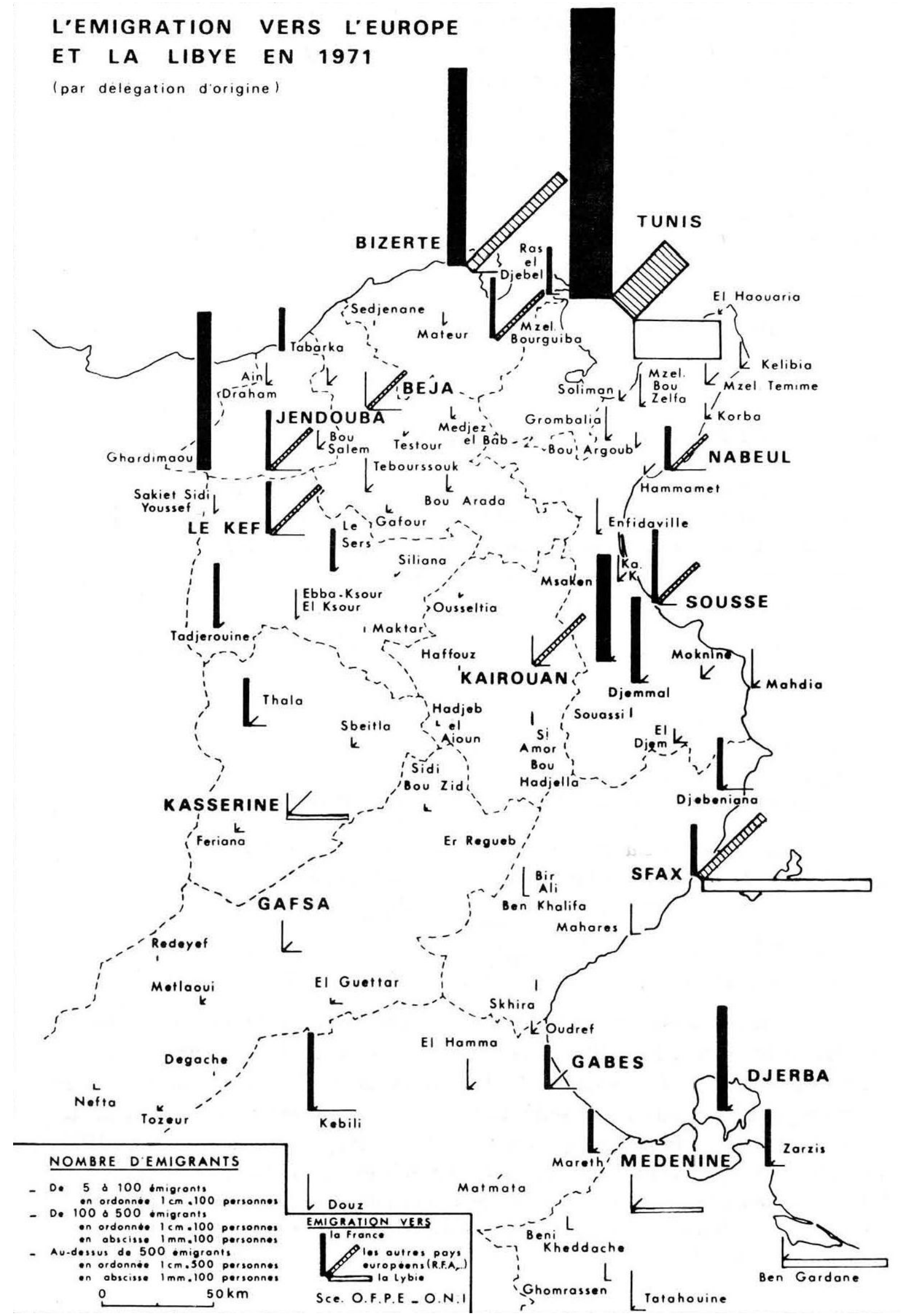

Figure 1 


\section{B - LES CARACTERES DEMOGRAPHIQUES}

Sur le plan démographique comme sur le plan professionnel, cette émigration ouvrière présente des caractères éminemment sélectifs. Elle concerne, tout d'abord, une population presqu'exclusivement masculine (4\% de travailleurs permanents féminins à l'entrée en France en 1970, et en 1971 - Source: Office National d'Immigration).

La répartition par tranche d'âge fait apparaître la prépondérance des départs entre 20 et 30 ans . En 1971

$\begin{array}{llll}\text { - } & \text { moins de } 20 \text { ans } & : & 10,6 \% \\ \text { - } & \text { moins de } 20-29 \text { ans } & : & 55,7 \% \\ \text { - } & \text { moins de } 30-39 \text { ans } & : & 24,4 \% \\ \text { - } & \text { plus de } 40 \text { ans } & : & 9,3 \% \text { (source : O.F.P.E.) }\end{array}$

Une tendance au rajeunissement se manifeste depuis quelques années, du moins dans le mouvement dirigé vers la France :

\begin{tabular}{rcccc} 
& 1967 & 1968 & 1970 & 1971 \\
moins de 25 ans & $29 \%$ & $35 \%$ & $38 \%$ & $44 \%$ \\
plus de 45 ans & $7 \%$ & $8 \%$ & $4 \%$ & $4 \%$ \\
& \multicolumn{4}{c}{ (source: } \\
O.N.I.)
\end{tabular}

Ce rajeunissement entraîne une augmentation de l'importance des célibataires (49 \% des travailleurs permanents entrés en France en 1967, 59 \% en 1970, 63 \% en 1971 - source: O.N.I.) mais on note parallèlement l'arrivée dans ce pays de femmes et d'enfants venant rejoindre les chefs de famille déjà installés. Près de 4000 personnes ont ainsi émigré vers la France en 1971, soit 32 \% de l'ensemble des Tunisiens contrôlés par l'O.N.I. contre 25 \% en 1970. Cette émigration familiale ne possède une certaine ampleur que vers la France, étant donné la politique très restrictive suivie par les autres pays, en ce domaine.

\section{C - LES CARACTERES PROFESSIONNELS}

La répartition par branches d'activité des travailleurs migrants fait apparaître en première place les emplois du bâtiment (28 \%) en deuxième l'industrie (17 \%) en troisième, l'agriculture (13 \%) l'hôtellerie (4 \%) mais il faut noter l'importance des emplois divers (34 \%) et les départs pour stages de formation ou de perfectionnement (2 \%).

On doit insister sur l'amélioration très nette de la qualification professionnelle obtenue depuis quelques années grâce aux efforts de l'O.F.P.E. Les statistiques françaises permettent de suivre ce progrès :

La proportion des manoeuvres est tombée de $58 \%$ en 1967 à $31 \%$ en 1971, alors que celles des ouvriers spécialisés et qualifiés est montée à $69 \%$ contre $42 \%$ en 1967. Le niveau de qualification est supérieur à celui des Marocains (57 \% de manoeuvres, en 1971) et des travailleurs portugais. Il est égal au niveau des travailleurs espagnols émigrés en France en 1971. Il semble que le degré de qualification des Tunisiens émigrant en France et en Libye soit plus élevé que ceux qui se dirigent vers la R.F.A. (52 \% de manœuvres en 1970) et vers les autres pays européens. 


\section{III - L'ORIGINE GEOGRAPHIQUE}

\section{A - LA REPARTITION REGIONALE}

La contribution des différentes régions tunisiennes à l'émigration n'est pas uniforme, des foyers d'émigration intense apparaîssent alors que de vastes régions ne réagissent guère à la stimulation migratoire.

La carte des départs en 1971 fait ressortir le déséquilibre entre la façade littorale orientale, de Bizerte à Jerda, dont la participation est largement prédominante, et l'intérieur, où les foyers migratoires sont plus rares.

Sur la bordure orientale, plusieurs foyers se distinguent très nettement : tout $d$ 'abord l'agglomération tunisoise avec près de 30 \% des départs, en 1971, puis le Sahel de Sousse et dans une moindre mesure celui de Sfax (20\% pour ces deux gouvernorats), la région de Médenine et plus faiblement celle de Gabès (13\%), le gouvernorat de Bizerte $(8 \%)$ et celui de Nabeul (5\%).

A l'intérieur, le principal foyer s'étend sur le Tell inférieur au contact avec la frontière algérienne (gouvernorat de Jendouba, et du Kef : 11 \% des départs en 1971).

Par contre, un vaste triangle central s'étend sur les gouvernorats de Béja, de Kairouan, de Kasserine et de Gafsa ne fournit à lui seul que $11 \%$ des partants, alors qu'il renferme le quart de la population tunisienne. Cette région à faible activité migratoire englobe aussi la partie Sud-Est des gouvernorats du Kef, de Béja, ainsi que l'arrière-pays sfaxien.

Cette répartition géographique de l'émigration tunisienne présente une certaine complexité due à l'interférence de nombreux facteurs d'ordre démographique, économique, sociologique et administratif. On doit se borner à énumérer quelques-uns de ces facteurs dont l'intervention est évidemment variable :

- distribution nationale de la population;

- possibilités et dynamisme de l'économie régionale, date de la rupture de l'équilibre démo-économique ;

- répartition et nature du sous-emploi selon le milieu d'origine (rural ou urbain);

- $\quad$ rapports avec les migrations internes et la polarisation de l'espace par les villes ;

- degré de cohération sociale pour la mise en place et la permanence des structures de circuits migratoires ;

- intervention des organismes administratifs pour la distribution des contrats de départ à l'étranger (offres anonymes).

\section{B - L'EMIGRATION URBAINE}

En prenant comme critère les délégations dont la population urbaine est supérieure à $50 \%$ et en y ajoutant les chefs-lieux de gouvernorats, on constate que les 2/3 des migrants de 1971 sont d'origine urbaine. Mais ce caractère de l'émigration est récent.

En effet, pendant la période qui a suivi l'indépendance, les villes, et surtout celles de la zone côtière ont bénéficié des principaux investissements privés et publics (construction, industries, 
tourisme), du développement des activités de services, ainsi que des emplois libérés par le départ de la population européenne et israëlite. Ceci explique que, selon le recensement, la majorité de ces villes connaissaient en 1966 une situation de l'emploi moins tendue que dans les campagnes et dans les régions de l'intérieur.

Mais, depuis quelques années, la poussée de l'urbanisation, le développement des migrations interrégionales, les difficultés de l'économie urbaine ont entraîné un renforcement de la pression migratoire dans les villes d'où les départs à l'étranger sont de plus en plus fréquents. Cette évolution n’est plus spécifique de la Tunisie, on la rencontre également en Algérie et au Mxroc.

L'origine socio-professionnelle des émigrants urbains est plus variée que dans l'émigration rurale . On distingue tout d'abord la masse des journaliers, sans aucune qualification et sans attache véritable avec le milieu urbain. Emigrants souvent amenés à la ville par l'exode rural, ils viennent des quartiers sous-intégrés et vivent précairement de petits métiers et d'emplois sporadiques. Ils partent comme simples manoeuvres.

Il y a ensuite les jeunes à la recherche de leur premier emploi dont une bonne partie vient de l'extérieur, c'est le cas de la capitale où le pourcentage des demandes d'emploi non satisfaites (premier emploi) est très supérieur à celui des autres gouvernorats. Une partie notable de ces jeunes sort des centres de formation professionnelle et de perfectionnement souvent implantés dans la banlieue des principales villes et dans les chefs-lieux de gouvernorat.

La main-d'oeuvre possédant déjà une certaine expérience professionnelle (ouvriers spécialisés et qualifiés) constitue enfin une part importante de cette émigration, nettement plus nombreuse que dans l'émigration rurale. On manque de statistiques pour apprécier cette caractéristique pour l'ensemble du mouvement migratoire urbain mais un sondage au quart effectué sur l'émigration vers la France en 1971 (60 \% de l'émigration globale) montre nettement que les gouvernorats les plus urbanisés, ceux de la zone côtière (Bizerte, Tunis, Nabeul, Sousse, Sfax) présentent les plus fortes proportions de travailleurs expérimentés et spécialisés, et l'éventail professionnel le plus varié. Les émigrants qui sont d'origine urbaine, le plus souvent locale mais aussi extérieure, peuvent être effectivement au chômage dans ces villes, mais ils peuvent aussi abandonner, en émigrant, un emploi permanent dans le secteur industriel et artisanal, selon les raisons précédemment évoquées. L'avantage des villes pour les travailleurs candidats à l'émigration est qu'ils peuvent s 'y familiariser avec les pratiques professionnelles modernes en manière de construction et de travaux publics beaucoup plus facilement que dans les campagnes. Il y a, en outre, dans certaines villes (comme Sfax) des traditions de travail artisanal ou ouvrier, qui donnent à leurs émigrants des qualités de plus en plus recherchées par les employeurs étrangers, au détriment de la main-d'œuvre sans expérience, ni qualification, originaire du milieu rural.

Mais tous les types de villes ne participent pas de façon égale à ce mouvement migratoire :

- les cités minières (Haut Tell, région phosphatière de Gafsa) connaissent très peu de départs pour l'étranger ;

- certaines agglomérations en cours d'urbanisation mais ayant gardé des liens étroits avec le milieu rural environnant constituent de véritables pôles d'émigration (notamment dans le Sahel central, à M'saken, Jemmal, Ksar Hellal) et dans le Sud (Zarzis) ; il y a là un phénomène en relation très étroite avec l'émigration rurale ;

- les chefs-lieux de gouvernorat ont une importance -sans cesse croissante, Tunis exceptée; ils totalisent en 197138 \% des émigrants urbains. Leur rang est dû à leur 
poids humain, à leur puissance d'attraction régionale mais aussi à l'installation des Bureaux Régionaux de l'Emploi et aux avantages pratiques qui en découlent pour l'émigration.

L'agglomération tunisoise tient une place essentielle dans l'émigration tunisienne puisqu'elle fournit $30 \%$ du total des partants et $44 \%$ des émigrants urbains. Il faut insister surtout sur le rôle de distributeur, de relais ou de tremplin migratoires qu'elle joue vis-à-vis des autres régions, presque $57 \%$ des émigrants proviennent des autres gouvernorats (cf. carte gvt. de départ, et gvt. de résidence). Les statistiques actuelles ne permettent pas de déterminer quelle est la géographie exacte de cette migration interne, on peut simplement constater que les gouvernorats de Béja, le Kef, Kairouan et Médenine connaissent la ponction la plus forte, proportionnellement au nombre de leurs ressortissants (émigrants nés dans un gouvernorat) . Ce rôle de tremplin migratoire joue notamment pour les jeunes à la recherche d'un premier emploi, et pour les travailleurs qualifiés.

\section{C - L'EMIGRATION RURALE}

Bien que l'émigration rurale soit minoritaire sur le plan statistique en 1971, elle reste néanmoins importante, en particulier par son rôle déterminant dans l'économie de certaines régions (Sud). D'ailleurs, en fondant l'analyse non plus sur les flux actuels mais sur l'ensemble de la population ayant émigré depuis 1956 et en y intégrant ces grosses agglomérations situées en milieu rural et vivant véritablement en symbiose avec lui, il est probable que le rapport est alors, inversé en faveur de l'émigration rurale.

Quel que soit le point de vue adopté, il est certain que cette émigration présente des caractères très différents de celle qui est issue des villes.

Les enquêtes directes effectuées dans les principaux foyers de départ montrent que le mouvement vers la France s'y est développé avec une certaine ampleur à une date plus ancienne que dans les villes. Il en est d'ailleurs de même en Algérie et au Maroc. Les départs ont commencé dans le Sud vers 1956, dans le Sahel en 1960 et à une date plus récente dans les régions du Kef et de Jendouba. Les régions fortement peuplées mais ne disposant que de ressources précaires et limitées (gouvernorats de Médenine et de Gabès , Sahel central) ont été plus affectées que d'autres par la crise agricole et la baisse de leur population agricole a été particulièrement forte (cf. tableau population active 1956-1966). Ce délestage a alimenté en partie les migrations internes mais aussi les départs vers l'étranger, surtout dans le Sud. En outre, la baisse de l'emploi agricole a été aggravée dans certaines régions par la régression des activités et des ressources complémentaires apportées traditionnellement par l'artisanat, dans le Sahel et à Jerba, ou par la petite pêche côtière (Jerba, Zarzis, Mareth, Maharès, Sahel de Sousse, et de Bizerte). La diffusion que l'on constate actuellement dans tout le milieu rural est un phénomène récent où intervient l'administration qui s'efforce de répartir les offres en fonction du sous-emploi régional et local. On comprend que par ses activités d'origine cette main-d'oeuvre rurale présente une formation et une qualification inférieure à celle des villes, le taux d'analphabétisme est d'ailleurs plus élevé.

Mais les faits sociologiques interviennent aussi car il est frappant de constater qu'une partie importante des émigrants ruraux viennent de gros pôles, tant dans le Sahel et surtout dans le Sud (Jerba, Zarzis, Tataouine, Mareth, Kébili). Ces pôles correspondent à des vieilles communautés sédentaires, caractérisées par un sens social très fort qui assure aux partants une certaine sécurité matérielle et morale tant dans le village d'origine vis-à-vis de la famille, que 
dans le lieu d'implantation où ils reçoivent l'aide de leurs compatriotes (logement - recherche d'un emploi - aide en cas de chômage ou de maladie). De cette cohésion migratoire découlent un certain nombre de caractères : concentration en gros foyers d'émigration, fort regroupement géographique à l'étranger, stabilité des courants migratoires - intensité des liens gardés avec le village d'origine - départs des éléments masculins et faiblesse de l'émigration familiale - régularité des retours. Ces traits sont des éléments de différenciation majeure avec l'émigration urbaine où les communautés ont souvent été dissociées par la migration interne, mais aussi avec les régions rurales fondées, autrefois ou encore aujourd'hui, sur l'élevage pastoral et qui ne possèdent pas une organisation sociale comparable. Ainsi s'explique à notre avis la différence majeure avec la région centrale correspondant aux steppes et aux basses plaines telliennes, où le problème du sous-emploi est cependant aigu, mais où l'absence de cohésion sociale a empêché jusqu'à maintenant l'établissement de véritables structures migratoires vers l'étranger.

\section{D - LA REPARTITION REGIONALE PAR PAYS}

L'émigration vers la France qui est encore majoritaire est nettement plus diffuse que les autres courants d'émigration. Elle possède des racines maintenant bien implantées en milieu rural notamment dans le Sud mais elle est alimentée plus largement que les autres par l'agglomération tunisoise qui relaie pour une forte part l'émigration rurale. Elle se maintient actuellement par les offres nominatives.

L'émigration vers la R.F.A. et les autres pays européens est beaucoup plus récente (1969). Le rôle de l'O.F.P.E. l'emporte très largement sur les démarches individuelles ; son origine géographique reste limitée aux villes et plus exactement aux chefs-lieux des gouvernorats.

Les départs vers la Libye ont connu une reprise depuis la ratification d'un nouvel accord de main-d'oeuvre en 1971. Elle est aussi le fait des chefs-lieux de gouvernorats, et plus spécialement de Tunis. L'intensité plus grande de mouvement dans la région sfaxienne et surtout dans la zone frontalière du Sud est due aux relations humaines et commerciales anciennes existant entre la Libye et ces deux régions.

\section{IV - CONSEQUENCES ET PERSPECTIVES DU MOUVEMENT MIGRATOIRE}

\section{A - LES CONSEQUENCES DEMOGRAPHIQUES}

L'augmentation des flux migratoires intervenue depuis 1956 a pour effet de freiner la croissance démographique. Le solde migratoire annuel en faveur de l'étranger (32 400 en 1971) représente de 20 à $25 \%$ de l'accroissement naturel annuel. Son influence sur la natalité est probable, compte tenu des caractères démographiques précédemment évoqués, mais elle reste encore à démontrer sur le plan statistique.

Son impact est plus facile à constater au niveau régional et il n'est pas douteux que les effets de l'émigration se conjuguent de façon notable à ceux des migrations internes. Ainsi en témoigne la diminution de la croissance démographique, voire même les régressions constatées en milieu rural surtout, mais aussi dans les villes du Sahel bizertin, du Sahel de Sousse et des gouvernorats du Sud (Gabès et Médenine). La carte de la population active 
1956-1966 montre bien l'évolution négative des gouvernorats de Sousse mais surtout de Gabès et de Médenine où la ponction migratoire est proportionnellement la plus forte.

\section{B - LES CONSEQUENCES ECONOMIQUES}

\section{LES RENTREES EN DEVISES}

Au niveau national, l'évolution de l'épargne transférée n'est pas toujours facile à établir car la plupart des émigrés rapportent avec eux de l'argent, différents produits (vêtements, matériel divers) et parfois aussi des véhicules à l'occasion des congés et des retours annuels.

Mais le bénéfice n'en est pas moins net et ne cesse de progresser fortement d'une année à l'autre.

\begin{tabular}{|c|c|c|c|c|}
\hline \multicolumn{5}{|c|}{ Recettes des revenus du travail (source B.C.T.) } \\
\hline 1963 & $:$ & $2400000 d$ & nars & \\
\hline 1964 & : & 2400000 & $"$ & \\
\hline 1965 & : & 3400000 & $"$ & \\
\hline 1966 & : & 4400000 & $"$ & \\
\hline 1967 & : & 6500000 & " & \\
\hline 1968 & : & 7300000 & $"$ & \\
\hline 1969 & : & 11400000 & $"$ & \\
\hline 1970 & : & 13000000 & " & dont $82 \%$ vient du travail des ouvriers émigrés \\
\hline 1971 & : & 18000000 & " & \\
\hline
\end{tabular}

L'émigration constitue actuellement une très importante source de rentrées de devises après le tourisme (32 millions de dinars) et le pétrole (25 millions de dinars) et ceci, sans contre-partie, à la différence du tourisme. Il faudrait cependant apprécier l'effet inflationniste de ces revenus extérieurs.

Sur le plan de l'emploi, les effets ne sont pas essentiels étant donné le volume global du sousemploi masculin et féminin. Mais l'influence est certainement sensible au niveau de l'emploi des jeunes jusqu'à l'âge de 30 ans, puisque près de 100000 travailleurs permanents ont ainsi quitté la Tunisie de 1964 à 1971 dont 63746 pour la France (Source O.N.I.). Certes, un certain pourcentage de ces émigrés est revenu définitivement en Tunisie mais le bilan général sur le plan de l'emploi reste très positif.

Au niveau régional, les conséquences n'apparaissent nettement que là où l'émigration a pris un caractère massif. La répartition des mandats postaux donne une idée assez exacte de la distribution des revenus provenant du travail à l'étranger. Selon le rapport de la B.C.T. en 1970, le Sud recevait la part la plus élevée de ces revenus (21 \% pour le gouvernorat de Médenine et $16 \%$ pour celui de Gabès). Cette priorité s'explique d'une part par le nombre total de travailleurs émigrés depuis 1956 mais aussi par la régularité plus grande de ces envois qui est le fait de cette émigration très attachée à sa région et possédant un sens aigu de ses obligations familiales et sociales. Tunis vient ensuite avec $19 \%$ puis Sousse (13\%). Bizerte (8 \%) et le Kef (5\%).

La répartition en volume des mandats envoyés de France et des autres pays européens par les travailleurs tunisiens (fig. 2) confirme dans l'ensemble la Îocalisation et la destination des flux migratoires en 1971 (carte 1), exception faite de la Libye avec laquelle il n'y avait pas à cette date de transfert par mandat postal. 


\section{REPARTITION DES MANDATS POSTAUX}

\section{EN PROVENANCE DE L'ETRANGER (1971)}

SCE PIT TINIS

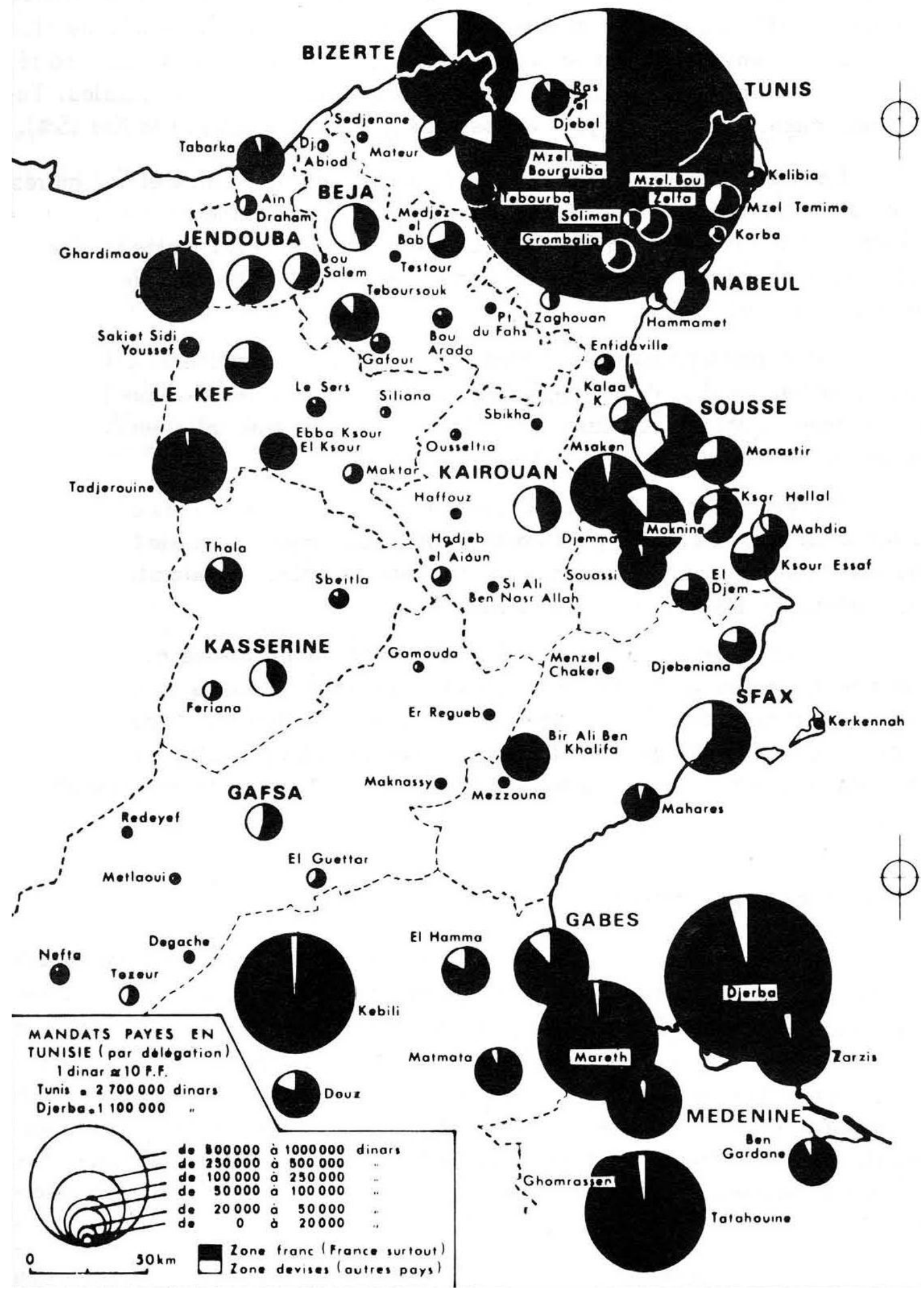


Les apports procurés par l'émigration sont proportionnellement plus forts en milieu rural où ils se combinent souvent avec d'autres revenus (agriculture, artisanat, pêche) que dans les villes où ils sont très inférieurs aux ressources procurées par les autres emplois.

L'argent gagné à l'étranger est utilisé pour les dépenses de consommation courante, et ensuite pour l'amélioration de l'habitat dont la transformation est la plus spectaculaire dans les principaux pôles d'émigration (M'saken, Tataouine, Mareth, Jerba, etc ...).

Les investissements productifs sont en revanche faibles ou nuls. L'argent s'investit peu dans l'agriculture (achat de terre, d'arbres ou de matériel agricole) rarement dans le tourisme, jamais dans l'industrie. Il est plus fréquent de le voir se diriger vers le tertiaire peu évolué où s'intègrent de préférence les émigrés de retour au pays (petit commerce, taxi, réparation automobile)

\section{C - LES PERSPECTIVES}

Sur le plan national, les perspectives d'emploi pour les années prochaines obligent à considérer la prolongation de l'émigration comme une nécessité absolue, un mal nécessaire, vu les difficiles conditions de travail et de vie des travailleurs expatriés. Mais se pose évidemment le problème de débouchés et de leur vulnérabilité en cas de ralentissement ou de crise économique dans les pays d'accueil. Ce qui justifie la politique officielle de diversification menée actuellement, mais on sait que l'évolution économique des pays industrialisés de l'Europe Occidentale est de plus en plus liée et interdépendante.

La France demeure le principal débouché mais l'émigration y a connu une baisse sensible en 1970 (11 070) : mais l'année 1969 était exceptionnelle (14 925) - ainsi qu'en 1971 : 9971 (20 $\%$ de moins que l'année précédente). Cette diminution est due à la mauvaise conjoncture qui règne actuellement dans ce pays où la baisse de l'immigration totale a été de $22 \%$ par rapport à l'année précédente. Cependant l'implantation solide réalisée maintenant et les qualités appréciées de la main-d'oeuvre tunisienne dans ce pays permettent d'escompter une stabilisation de cette évolution, à un niveau qui reste d'ailleurs supérieur à celui de la période 1966 - 1968 (6 000 en moyenne) (Source : O.N.I.).

Les départs vers l'Allemagne Fédérale ont connu aussi une diminution en 1971 : 2769 départs contre 4990 en 1970 mais le déficit a été comblé par le développement de l'émigration vers les autres pays (Libye, Pays-Bas, Autriche, Suisse, Belgique) à la suite des différents accords de main-d'oeuvre. La reprise économique amorcée en R.F.A. au mois de mars 1972 et l'accroissement de l'immigration qui en a résulté permettent d'espérer une reprise des départs vers ce pays.

A ce problème de la sensibilité à la conjoncture économique et la vulnérabilité qui en résulte pour l'économie nationale mais surtout régionale. Il faut aussi ajouter celui du départ de la main-d'oeuvre qualifiée et des difficultés qui en résultent pour certaines entreprises nationales. 


\section{TRAVAUX RECENTS SUR L'EMIGRATION TUNISIENNE}

1. ABDEDDAIEM (Ali) - Changement social chez les travailleurs tunisiens de Belleville. Mémoire Ecole pratique des Hautes Etudes - 1971.

2. BEN SASSI (T.) - Les travailleurs tunisiens dans la région tunisienne - Hommes et Migrations, $\mathrm{n}^{\circ} 109$ - 1968 - $115 \mathrm{p}$. - 1 carte.

3. HARGUEM (Ahmed) - Les ouvriers tunisiens. Organisation administrative et politique - Mémoire de 3.e cycle dactylographié - Institut d'Etudes de Développement économique et social - Paris - 1971 - $194 \mathrm{p}$.

4. MHILI (Ali) - L'amicale des travailleurs tunisiens en France - Mémoire Ecole Pratique des Hautes Etudes - Paris - 1969.

5. SIMON (G.) - L'émigration et son rôle dans le développement régional du Sud Tunisien - 2e colloque de géographie Maghrébine - Sept . 1970 - à paraître dans Annales algériennes de géographie.

6. SIMON (G.) et NOIN (D.) - La migration maghrébine vers l'Europe occidentale Cahiers d'Outre-Mer - nº9 - juillet-septembre 1972 - pp.241-276 - 7 figures.

7. ZAMITI (M .) - Migrations et cultures. Les ouvriers tunisiens en France - Thèse de 3e cycle - ronéotée - Paris - 1971 - 280 p.

\section{NOTES}

(1) Cette analyse s'insère dans un rapport sur « l'Armature urbaine de la Tunisie », réalisé par le groupe Huit (Tunis) pour le compte de l'Aménagement du Territoire. Tunis. 1973.

(2) SIMON (G.) et NOIN (O.) n5. p.248. 